\title{
Mediation analysis with a time-to-event outcome: a review of use and reporting in healthcare research
}

Lauren Lapointe-Shaw ${ }^{1,2,3^{*}} \mathbb{D}$, Zachary Bouck ${ }^{4}$, Nicholas A. Howell ${ }^{2,3,5}$, Theis Lange ${ }^{6,7}$, Ani Orchanian-Cheff ${ }^{8}$, Peter C. Austin ${ }^{2,3}$, Noah M. Ivers ${ }^{2,3,4,9}$, Donald A. Redelmeier ${ }^{1,2,3}$ and Chaim M. Bell ${ }^{1,2,3,10}$

\begin{abstract}
Background: Mediation analysis tests whether the relationship between two variables is explained by a third intermediate variable. We sought to describe the usage and reporting of mediation analysis with time-to-event outcomes in published healthcare research.

Methods: A systematic search of Medline, Embase, and Web of Science was executed in December 2016 to identify applications of mediation analysis to healthcare research involving a clinically relevant time-to-event outcome. We summarized usage over time and reporting of important methodological characteristics.

Results: We included 149 primary studies, published from 1997 to 2016. Most studies were published after 2011 ( $n=110,74 \%$ ), and the annual number of studies nearly doubled in the last year (from $n=21$ to $n=40$ ). A traditional approach (causal steps or change in coefficient) was most commonly taken $(n=87,58 \%)$, and the majority of studies ( $n=114,77 \%$ ) used a Cox Proportional Hazards regression for the outcome. Few studies $(n=52$, $35 \%)$ mentioned any of the assumptions or limitations fundamental to a causal interpretation of mediation analysis.

Conclusion: There is increasing use of mediation analysis with time-to-event outcomes. Current usage is limited by reliance on traditional methods and the Cox Proportional Hazards model, as well as low rates of reporting of underlying assumptions. There is a need for formal criteria to aid authors, reviewers, and readers reporting or appraising such studies.
\end{abstract}

Keywords: Mediation, Indirect effect, Counterfactuals, Reporting, Mediation analysis, Survival, Time-to-event, Methodology

\section{Background}

Mediator variables lie along the causal pathway between an independent and dependent variable, explaining all or part of the effect of the independent variable on the dependent variable [1]. While mediation analysis has been prominently featured in social science research, this methodology is now gaining popularity in healthcare research. It is used primarily for two purposes: to understand how certain relationships (including treatment effects) occur, and to identify possible targets for future

\footnotetext{
* Correspondence: lauren.lapointe.shaw@mail.utoronto.ca ${ }^{1}$ Department of Medicine, University of Toronto, Toronto, Canada ${ }^{2}$ Institute for Health Policy, Management and Evaluation, University of Toronto, Toronto, Canada

Full list of author information is available at the end of the article
}

interventions [1]. A test of mediation examines whether the effect of the independent variable $(\mathrm{x})$ on the dependent variable (y) occurs via a third, intervening variable $(\mathrm{z})$ (see Figs. 1, 2). This basic structure - referred to as a single-mediator model - can be expanded to include additional considerations such as multiple mediators and moderated mediation [2-5].

The causal interpretation implicit in any mediation analysis rests on a number of untestable assumptions, which are often underreported in published research $[6,7]$. In particular, the sequential ignorability assumption states that there is no unmeasured confounding of the exposure-mediator, mediator-outcome, or exposure-outcome relationships [8]. Furthermore, there must be no confounders (measured or unmeasured) of

(c) The Author(s). 2018 Open Access This article is distributed under the terms of the Creative Commons Attribution 4.0 International License (http://creativecommons.org/licenses/by/4.0/), which permits unrestricted use, distribution, and reproduction in any medium, provided you give appropriate credit to the original author(s) and the source, provide a link to the Creative Commons license, and indicate if changes were made. The Creative Commons Public Domain Dedication waiver (http://creativecommons.org/publicdomain/zero/1.0/) applies to the data made available in this article, unless otherwise stated. 


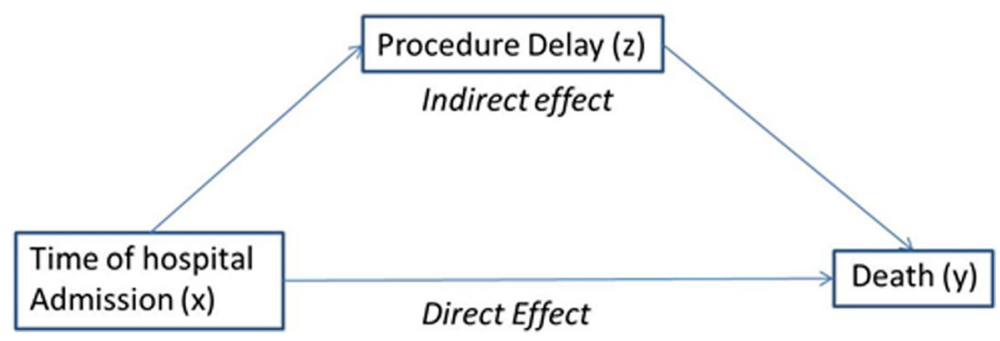

Fig. 1 Causal diagram depicting the relationship between independent $(x)$, dependent $(y)$, and mediator $(z)$ variables

the mediator-outcome that depend on the exposure [9-11]. While these assumptions can theoretically be satisfied by random allocation, it is not possible to randomise both exposure and mediator [12]. As a consequence, some suggest that any mediation analysis be accompanied by sensitivity analyses to investigate the robustness of findings to violations of this crucial assumption [8]. Furthermore, tests of mediation assume that the mediator has been appropriately defined and measured [13]. In addition to these fundamental assumptions, the temporal sequence of independent variable, mediator, and dependent variable should support the argument for causation $[14,15]$.

Traditional methods of mediation analysis include fulfilling a series of stepwise criteria (causal steps), as proposed by Baron and Kenny in 1986 [16]. To quantify the degree of mediation, simple formulas combine parameter estimates obtained from a series of regressions $[1,17,18]$. The resulting difference and product tests were originally intended for linear relationships with continuous outcomes such as blood pressure, but have been adapted for binary outcomes such as mortality. Unfortunately these methods are ill-adapted to non-normally distributed continuous and/or censored variables, such as time-to-event outcomes [14].

Mortality and survival time are a major focus in healthcare research. Survival analysis allows investigators to study these important outcomes with appropriate consideration for variable follow-up times, censoring, and competing risks. Cox Proportional Hazards (PH)

\section{MEDIATION ANALYSIS IN HEALTHCARE RESEARCH: AN EXAMPLE}

Several studies have found that patients admitted to hospital on weekends experience higher rates of mortality than patients admitted on weekdays. One possible explanation for this difference is delayed procedures for weekend patients due to reduced staffing at off-hours. Testing this hypothesis would require the use of mediation analysis, a method that seeks to explain how an exposure leads to an outcome. In our example mediation model (Figure 1), it is hypothesized that weekend admission may lead to increased mortality directly or indirectly through procedural delay.

Use of mediation analysis requires that we account for baseline differences between weekend and weekday patients that might affect their procedural timing or mortality in hospital (e.g. severity of illness). Of note, neither timing of hospital admission nor procedural delay can be randomised, thus other methods (such as regression) must be used to account for such differences.

Further, there should be no confounding by factors associated with both procedural delay and mortality that would also be affected by weekend admission. For example, if the skill of diagnosing physicians differed between weekends and weekdays and was also associated with both procedural delay and mortality, this would bias the results of mediation analysis. For this reason, it is important that all possible mediators (e.g. diagnosing physician skill in this case) be included in the model. The indirect effect through a combined mediator can then be measured.

We must also ensure that procedural delay is accurately measured, and captures only care that occurs after hospital admission. If procedural delay is measured in days, rather than minutes, this would bias the measurement of indirect effects toward the null. If procedural delay is measured from start of symptoms, rather than following hospital admission, this would raise the possibility that procedural delay is a confounder of the exposure-outcome relationship, rather than a mediator. Hospital admission, procedure timing, and mortality must be separate and sequential in time to support a causal interpretation of mediation analysis.

Fig. 2 An example of mediation analysis in healthcare research 
regression is commonly used for such analyses, yet its use in mediation analysis poses some important challenges. The semi-parametric Cox model builds on proportionality of the hazards. Proportionality is violated when adding an additional (mediator) variable to a correctly specified Cox regression model. This addition could shift the baseline hazards up or down, rather than only altering the slope of the hazard function [19]. Statisticians term this phenomenon the "non-collapsibility" of the hazard ratio [20]. As a result, parameter estimates obtained with and without a mediator cannot be meaningfully compared as they might be in a linear model $[21,22]$. This problem is exacerbated as the outcome frequency rises. Thus, use of $\mathrm{Cox} \mathrm{PH}$ regression to approximately estimate indirect effects via difference or product of coefficients rests on the assumption that the outcome is rare [21]. Parametric survival (including accelerated failure time) and additive hazard models do not have this limitation [14, 21]. These models provide readily interpretable outcome measures (expressed as hazard ratios or differences), yet they are less familiar to clinical researchers than the popular Cox model [14].

Path analysis provides another possible approach, and allows for modelling of the relationships between a large number of confounding and mediator variables [23]. Structural Equation Modelling (SEM) is derived from path analysis, and incorporates latent variables, allowing uncertainty of variable measurement to be incorporated into the analysis [23]. Using SEM and path analysis, relationships can be deconstructed into subcomponents and indirect effects obtained [24]. Although these models depend on linearity of relationships, time-to-event outcomes can be modelled in SEM and path using discrete time survival analysis or dynamic path analysis, wherein the follow-up period is broken down into short time intervals [25-28]. Such methods allow mediation effects to be expressed as hazard ratios and hazard differences, respectively. Drawing conclusions based on results of SEM and path analysis depends on adequate linear model specification, and that all included variables are free of unmeasured confounding [29].

As a result of the linearity assumptions inherent to previous methods of mediation analysis, alternative methods have been sought. The counterfactual or potential outcomes approach evolved more recently from the literature on causal inference [30]. In this framework, mediation analysis is treated as a problem of missing data, and observed and unobserved potential outcomes are modelled. This flexible approach can accommodate any data distribution, and be applied to any type of mediator or outcome variable, including time-to-event [8]. In addition to meeting the assumptions underlying a causal interpretation of mediation analysis, implementation of this approach requires meeting the assumptions inherent to any selected models. Within the counterfactual framework, additive hazard, parametric survival and marginal structural models also allow for measurement of indirect effects, without the limitation to rare outcomes [31].

While the above approaches offer a range of strategies to address mediation analysis with a time-to-event outcome, some require advanced statistical coding, or at least an understanding of counterfactual concepts. While mediation analysis is increasingly utilized, we do not know how healthcare researchers have addressed this problem. Although others have described the recent reporting of causal mediation analysis, they have not examined practices specific to time-to-event outcomes, nor have they described temporal trends in the use of these methods [6, 7]. We sought to evaluate the usage and reporting of mediation analysis with time-to-event outcomes in all published healthcare research.

\section{Methods}

\section{Systematic search and screening}

A systematic and sensitive search strategy, developed with a research librarian (AOC), was used to identify published articles employing mediation analysis with a time-to-event outcome. The search strategy was initially developed for Ovid Medline, and then customized for use in the other databases. At the time of the search, specific subject headings for mediation analysis and time-to-event were unavailable in the databases used. As a result, the strategy was devised using an extensive list of appropriate text words and phrases mined from sample articles and through input from subject specialists on the team. Ovid Medline, Ovid Medline Epub Ahead of Print and In-Process \& Other Non-Indexed Citations, and Ovid Embase were searched from inception to date of search. All searches were executed between December 9th and 12th, 2016. No limits for date were applied and animal-only studies were excluded where applicable. Book and conference materials were also excluded from Embase. In addition, cited reference searches were conducted in the Web of Science Core Collection for any articles citing one of five highly cited and relevant methodological articles [8, 31-34] (see Additional file 1 for details of search).

Studies relating to human healthcare, with an empiric application of mediation analysis and a clinically relevant time-to-event outcome, were selected for inclusion. Since we were most interested in how a non-specialist healthcare researcher applied the methodology, theoretical papers with an illustrative application were excluded. Review articles were manually searched for relevant primary studies.

Inclusion criteria were pre-specified and refined after pilot screening of 10 full-text articles. Specifically, 
inclusion criteria were refined to include a formal test of mediation, in the form of meeting specific listed criteria (e.g. directly cite Baron and Kenny or describe causal steps methodology), a statistical test with a $p$-value, or a measurement of indirect effect/proportion mediated. This was necessary as many studies did not set out to assess mediation, but mentioned it as a possible explanation for weakening of an observed association upon the introduction of other variables.

All eligible abstracts were screened in duplicate by LLS and ZB. Abstracts deemed eligible by either LLS or $\mathrm{ZB}$ were included for full-text review. All full texts were screened by LLS. Uncertainty in study inclusion or extraction was addressed by discussion with TL, a methodological expert. NAH performed duplicate full-text screening and extraction of a $10 \%$ random sample $(n=$ 33 ) in order to assess reproducibility.

Duplicate screening showed 82\% agreement, Cohen's kappa was 0.63 (95\% CI 0.36-0.89). Disagreements related to the relevance of a clinical outcome (sick leave, $n=1$, study was included) and whether a formal test of mediation was described ( $n=5$, all excluded). Though these five excluded studies did not explicitly state how they assessed mediation or indirect effects, they appeared to use the following strategies: partial causal steps $(n=1)$, change in coefficient $(n=4)$. They all used Cox PH models for the outcome, and none mentioned any of the assumptions fundamental to mediation analysis.

\section{Extraction}

The criteria for extraction were developed in consideration of the STROBE statement [35], existing systematic reviews of mediation analysis $[6,7,36]$, and methodological concerns unique to time-to-event outcomes.

After a pilot extraction from 10 full-text articles, extraction criteria were refined and all extraction performed by LLS. Where studies included a methodological supplement for mediation details, these were also reviewed for relevant information. The results of duplicate extraction from a $10 \%$ random sample of included studies are presented in Table 1 . The criteria tested for inter-rater reliability were pre-specified based on their importance. Estimates of Cohen's kappa (with 95\% confidence intervals) were obtained using the "kappa2" function in the "irr" package in R [37].

We extracted information on study characteristics including methodological approach to mediation analysis, statistical analysis, assumptions addressed, and measures reported. Results are presented as counts and frequencies for categorical or binary characteristics, and as median and interquartile range for study sample size.

As suggested by a peer reviewer, we added selected comparisons of studies published before or after 2013 .
Comparisons were made with the Chi-square test, with $p<0.05$ defined as significant; Fischer's exact text was used for comparisons where frequencies of 0 (empty cells) were reported.

\section{Results}

Our search yielded 1991 unique abstracts, of which 321 were selected for further review (see Fig. 3). Of these, 8 were excluded as they did not relate to human healthcare, 110 because they did not include mediation criteria, test, or measurement of the indirect effect/proportion mediated. Another 12 were excluded because they did not include a clinically meaningful outcome, and 41 because the outcome of mediation analysis was not time-to-event. Further, one full text could not be reviewed as it was in Arabic. This left 149 studies eligible for extraction (see Additional file 2 for the list of included studies).

The earliest study was published in 1997, and there were fewer than 10 studies per year up to 2011. There were 110 included studies (74\%) published in 2012 or later, and the number of studies nearly doubled from 2015 to 2016 ( $n=21$ in 2015, $n=40$ in 2016, see Fig. 4). Over half of included studies had a first author based in the United States $(n=77,51 \%)$, and 82 were from North America (55\%). Otherwise, 55 (37\%) publications originated in Europe, 5 (3\%) were from Asia, 5 (3\%) from Australia, $1(<1 \%)$ was from Israel and $1(<1 \%)$ from Brazil. Sixty-four individuals were listed as an author on more than one included study. The number of studies per author ranged from 1 to 6 , with 8 individuals listed on 5 or more studies. Included studies most commonly came from journals in the areas of epidemiology $(n=37,25 \%)$, psychology/psychiatry $(n=19,13 \%)$, cardiology $(n=17,11 \%)$, oncology $(n=13,9 \%)$, and general medicine $(\mathrm{n}=13,9 \%)$. Eleven studies $(7 \%)$ were published in high impact journals (impact factor 10 or greater) [38].

Mediation analysis was in most cases $(n=80,54 \%)$ not the primary study aim, and was frequently an exploratory analysis $(n=74,50 \%$, see Table 2$)$. Many studies $(n=76,51 \%)$ tested multiple mediators. The most commonly tested mediators were psychological or psychiatric $(n=32,21 \%)$, physiologic parameters $(n=34$, $23 \%)$ or lifestyle factors $(n=31,21 \%)$. The majority of mediators were continuous $(n=60,40 \%)$ or binary $(n=56$, $38 \%$ ) variables. The most common outcome was the onset of a new medical condition or exacerbation of an existing condition $(n=68,46 \%)$. A causal diagram was included in a third $(n=59,40 \%)$ of studies. Results supporting a mediation model were reported in 130 studies (87\%), and 19 studies (13\%) reported that all tested mediators either did not meet criteria or were statistically not significant. Sixty-four studies (43\%) reported mixed results (both 
Table 1 Agreement on important characteristics, at re-extraction of a 10\% random sample of included studies

\begin{tabular}{|c|c|}
\hline Characteristic & Unweighted Cohen's Kappa (95\% Cl) \\
\hline Funding source & $0.75(0.5-1)$ \\
\hline Study Design & $0.65(0.02-1)$ \\
\hline Type of analysis (confirmatory/hypothesis-based versus exploratory) & $0.14(0-0.5)$ \\
\hline Mediation analysis is primary aim of study & $0.52(0.12-0.92)$ \\
\hline Causal diagram included & $1(1-1)$ \\
\hline Sample size & $0.75(0.55-0.96)$ \\
\hline Power/sample size calculation included & 0 (unable to estimate, too infrequent) \\
\hline Method of mediation analysis & $0.84(0.83-1)$ \\
\hline Type of time-to-event model & $0.91(0.75-1)$ \\
\hline Competing risks considered & 0 (unable to estimate, too infrequent) \\
\hline If clustering of data, was this addressed in the analysis? & $0.61(0.15-1.0)$ \\
\hline Outcome frequency $>=10 \%$ & $0.79(0.54-1)$ \\
\hline Rare outcome limitation for Cox model mentioned & Unable to estimate, all false (100\% agreement) \\
\hline Temporal separation clearly defined & $0.76(0.47-1)$ \\
\hline No unmeasured confounding of exposure/outcome & $0.82(0.49-1)$ \\
\hline No unmeasured confounding of mediator/outcome & $0.85(0.57-1)$ \\
\hline No unmeasured confounding of exposure/mediator & $0.6(0.13-1)$ \\
\hline No exposure-dependent confounding of mediator-outcome & $0.64(0-1)$ \\
\hline Accurate measurement of mediator & $0.65(0.32-0.99)$ \\
\hline Interaction between exposure and mediator considered/tested & $0.6(0.19-1)$ \\
\hline Was a method used to address confounding of exposure or mediator? & N/A (100\% used regression for both exposure and mediator, 100\% agreement) \\
\hline Sensitivity analysis relating to mediation analysis & $0.44(0.05-0.75)$ \\
\hline Measures reported-indirect effect & $0.76(0.46-1)$ \\
\hline Measures reported-proportion mediated & $0.86(0.61-1)$ \\
\hline Precision estimate for indirect effect & $1.0(\mathrm{~N} / \mathrm{A})$ \\
\hline Precision estimate for proportion mediated & $0.77(0.34-1)$ \\
\hline
\end{tabular}

significant and not significant) for the various mediators being tested.

The most common method used for mediation analysis was comparing coefficients (henceforth known as "change in coefficient") before and after a mediator was introduced into an exposure-outcome regression model without testing the other relationships included in the causal steps approach $(n=46,31 \%)$. Other commonly used methods included causal steps $(n=41,28 \%)$, counterfactuals $(n=32,21 \%)$ and SEM or path analysis $(n=23,15 \%)$. Studies published prior to 2010 predominantly featured causal steps and SEM/path approaches. After 2011, there was increased use of counterfactuals, change in coefficient, and causal steps methods of mediation analysis (see Fig. 5).

The majority $(n=136,91 \%)$ of included studies described their funding source. In most cases $(n=113$, $76 \%)$ this was governmental. The majority of included applications were cohort studies $(n=131,88 \%)$. Most studies $(n=112,75 \%)$ did not report exposure, mediator and outcomes that were clearly separated and sequential in time. Most commonly, overlap occurred in measurement of exposure and mediator $(n=89,60 \%)$. The most common method used to deal with confounding of exposure and mediator was regression $(n=137,92 \%$ for exposure; $n=138,93 \%$ for mediator). Most studies did not mention any of the assumptions underlying mediation analysis $(n=97,65 \%)$, a third $(n=52,35 \%)$ mentioned at least one assumption, and eight (5\%) mentioned all the assumptions. Among studies with a primary aim to assess mediation $(n=69), 33(48 \%)$ mentioned one or more assumption, and six $(9 \%)$ mentioned all assumptions. Sensitivity analysis relating to mediation analysis was included in 25 studies (17\%).

Of 105 studies with outcomes other than all-cause mortality, four (4\%) included consideration of competing risks. Of 35 studies with possible clustering of data in the exposure or mediator, 19 (54\%) addressed this in their analysis. A third of studies $(n=46,31 \%)$ mentioned or tested for interaction between exposure and mediator. 


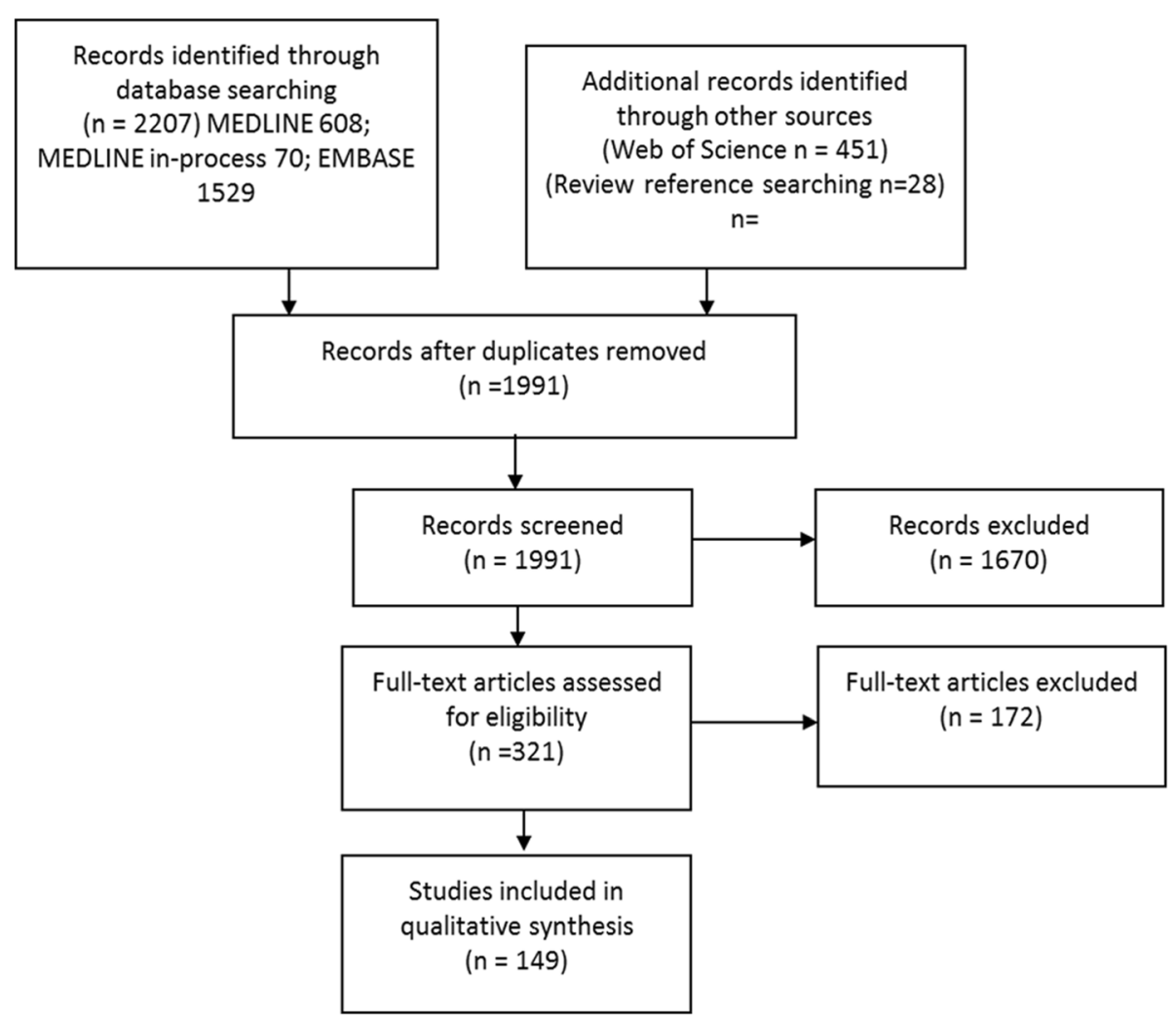

Fig. 3 PRISMA Flow Diagram

Sample sizes ranged from 23 to 2,940,453, with 10 studies (7\%) reporting sample sizes below 200. A single study included a sample size calculation, in this case for the association between the exposure and mediator [39], and another three studies discussed power and sample size as they relate to mediation. Software packages specifically used for mediation were mentioned in 32 studies (21\%).

Indirect effect was reported in 55 studies (37\%), proportion mediated in 83 studies (56\%); 38 studies

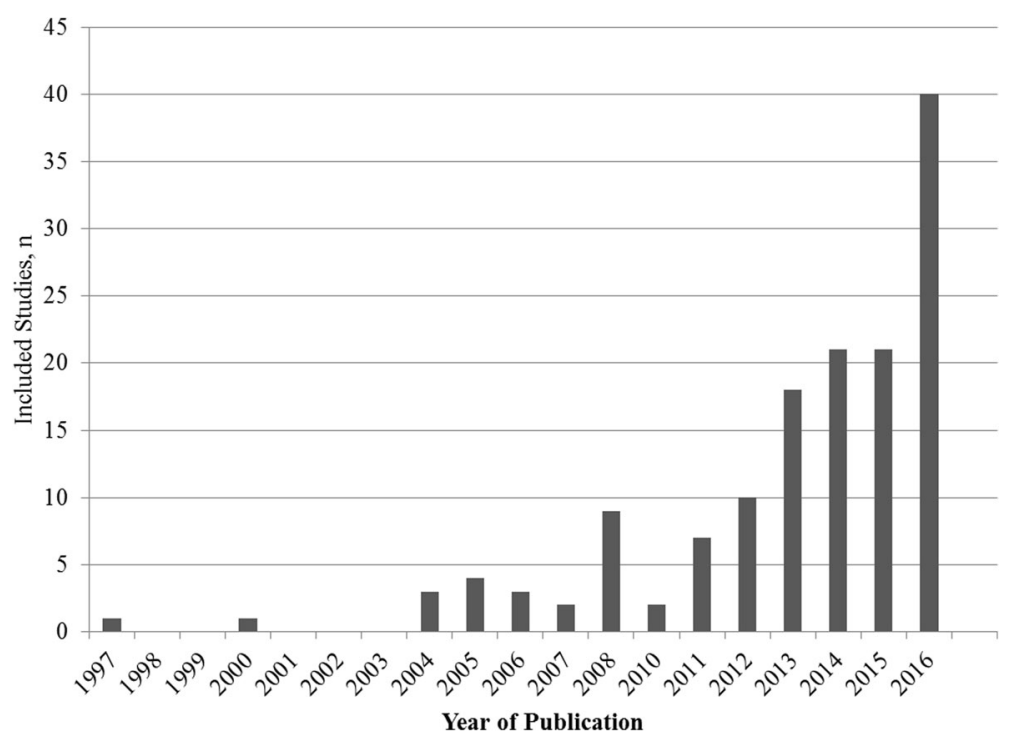

Fig. 4 Included studies, by year of publication 
Table 2 Characteristics of mediation analyses with time-to-event outcome in healthcare research, $n=149$

\begin{tabular}{ll}
\hline Included study characteristic & Result \\
\hline
\end{tabular}

Funding source, $n$ (\%)

Government

$37(25)$

Foundation

Hospital

Industry

Professional association

None stated

$13(9)$

Study design, $n$ (\%)

Cohort

Randomised Controlled Trial

Case-cohort

Case control

Cross-sectional

$1(<1)$

Type of analysis, $n(\%)$

Confirmatory/Hypothesis-based

$72(48)$

Exploratory

$74(50)$

Not able to infer

Mediation analysis is primary aim of study, $n$ (\%)

69 (46)

Multiple mediators tested, $n$ (\%)

$76(51)$

Type of mediator, $n$ (\%)

Continuous

$60(40)$

Binary

Categorical

$25(17)$

Interval/Ordinal

$25(17)$

Latent

Most common content of mediator ${ }^{\mathrm{a}}, n(\%)$

Physiologic (e.g. blood pressure, heart rate, weight)

Psychological/psychiatric

$34(23)$

$32(21)$

Lifestyle (e.g. alcohol, smoking, nutrition, exercise, sleep)

Biomarker (blood test results)

Health

Comorbidity

Treatment

Functioning

Socioeconomic

Environment

Reproductive

Most common outcomes, $n$ (\%)

New medical condition or exacerbation

of an

68 (46)

existing condition

All-cause mortality

$48(32)$
Table 2 Characteristics of mediation analyses with time-to-event outcome in healthcare research, $n=149$ (Continued)

\begin{tabular}{ll}
\hline Included study characteristic & Result \\
\hline Cause-specific mortality & $21(14)$ \\
Disability or sick leave & $6(4)$ \\
Causal diagram included, $n(\%)$ & \\
Causal steps/change in coefficient $(n=87)$ & $22(25)$ \\
Counterfactuals $(n=32)$ & $16(50)$ \\
SEM/path $(n=23)$ & $18(78)$ \\
Product of coefficients $(n=6)$ & $3(50)$ \\
Cannot infer $(n=1)$ & $0(0)$ \\
Sample size, median (IQR) & $3345(637-16,061)$
\end{tabular}

Power/sample size, $n(\%)$

Calculation $1(<1)$

Consideration 3 (2)

Method of mediation analysis, $n$ (\%)

Causal steps, including Baron-Kenny 41 (28)

Change in coefficient in a single regression 46 (31)

Counterfactuals $32(21)$

SEM/path $23(15)$

Product of coefficients 6 (4)

Cannot infer $\quad 1(<1)$

Statistical tests for no mediation/indirect effect, $n$ (\%)

Sobel 7 (5)

Other product test 14 (9)

Difference test 2 (1)

Z-test of mediated proportion $\quad 1(<1)$

Joint significance test $\quad 1(<1)$

Olaf \& Finn test $\quad 1(<1)$

Type of time-to-event model, $n$ (\%)

Cox proportional hazard 114 (77)

Additive hazard $10(7)$

Linear $\quad 7$ (5)

Discrete time survival model 6 (4)

Failure time/parametric survival 5 (3)

Marginal structural model 3 (2)

Log linear Poisson $\quad 1(<1)$

Quantile regression $\quad 1(<1)$

Cannot infer $\quad 5$ (3)

Specific mediation software mentioned, $n$

Causal steps/change in coefficient

SAS "mediate" macro 2

PRODCLIN

Counterfactuals

$\mathrm{R}$

R "mediation"

8

2 
Table 2 Characteristics of mediation analyses with time-to-event outcome in healthcare research, $n=149$ (Continued)

\begin{tabular}{ll}
\hline Included study characteristic & Result \\
\hline SAS & 1 \\
SAS "mediate" macro & 1 \\
STATA "medeff" & 1 \\
SEM/path & \\
Mplus & 13 \\
SAS & 1 \\
STATA mediation package & 1 \\
LISREL & 1 \\
Competing risks considered, $n(\%)$ & $4(3)$
\end{tabular}

If clustering of data, was this addressed in the analysis? $n$ (\%)

$$
\begin{aligned}
& \text { Not multilevel } \\
& \text { Yes } \\
& \text { No } \\
& \text { Cannot determine } \\
& \text { Cox models, outcome frequency }{ }^{\mathrm{b}}, n(\%) \\
& >\text { or equal to } 5 \% \\
& >\text { or equal to } 10 \%
\end{aligned}
$$$$
114
$$

Rare outcome limitation for Cox model mentioned ${ }^{\mathrm{b}}, \quad 8$ (7) $n(\%)$

Temporal separation clearly defined, $n$ (\%)

Yes

Overlap exposure and mediator

Overlap mediator/outcome

Cannot determine

Acknowledged as a limitation

Mediation assumptions (or limitation) stated, $n$ (\%)

No unmeasured confounding of exposure/outcome

No unmeasured confounding of

mediator/outcome

No unmeasured confounding of

exposure/mediator

No exposure-dependent confounding of mediator-outcome

Accurate measurement of mediator

Interaction between exposure and mediator considered/tested, $n$ (\%)

Method to address confounding of exposure (more than one can be used), $n$ (\%)

Regression/modelling

Stratification/restriction

Randomisation

None

Method to address confounding of mediator (more than one can be used), $n$ (\%)

Regression/modelling
Table 2 Characteristics of mediation analyses with time-to-event outcome in healthcare research, $n=149$ (Continued)

\begin{tabular}{ll}
\hline Included study characteristic & Result \\
\hline Weighting & $13(9)$ \\
Stratification/restriction & $13(9)$ \\
Matching & $1(<1)$ \\
None & $10(7)$
\end{tabular}

Sensitivity analysis related to mediation analysis, $n$ (\%)

Any

Confounding

Accurate measurement/specification of mediator

Temporal sequence assumption

Testing a combined mediator or all mediators in

$5(3)$ same model

Interaction/moderation

Measures of mediation reported, $n$ (\%)

Causal steps/change in coefficient method $(n=87)$

Indirect effect

Proportion mediated

$52(60)$

Counterfactuals $(n=32)$

Indirect effect

Proportion mediated

SEM/path $(n=23)$

Indirect effect

Proportion mediated

Other $(n=7)$

Indirect effect

3

Proportion mediated

4

Measures of precision reported, $n$ (\%)

Causal steps/change in coefficient $(n=87)$

Indirect effect confidence interval

Proportion mediated confidence interval

Statistical test $\mathrm{p}$-value or equivalent

Counterfactuals $(n=32)$

Indirect effect confidence interval

Proportion mediated confidence interval

SEM/path $(n=23)$

Indirect effect confidence interval

Proportion mediated confidence interval

Statistical test $p$-value or equivalent

Other $(n=7)$

Indirect effect confidence interval 3

Proportion mediated confidence interval 3

Statistical test $\mathrm{p}$-value or equivalent

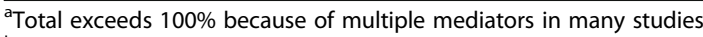
${ }^{b}$ Denominator is 114 


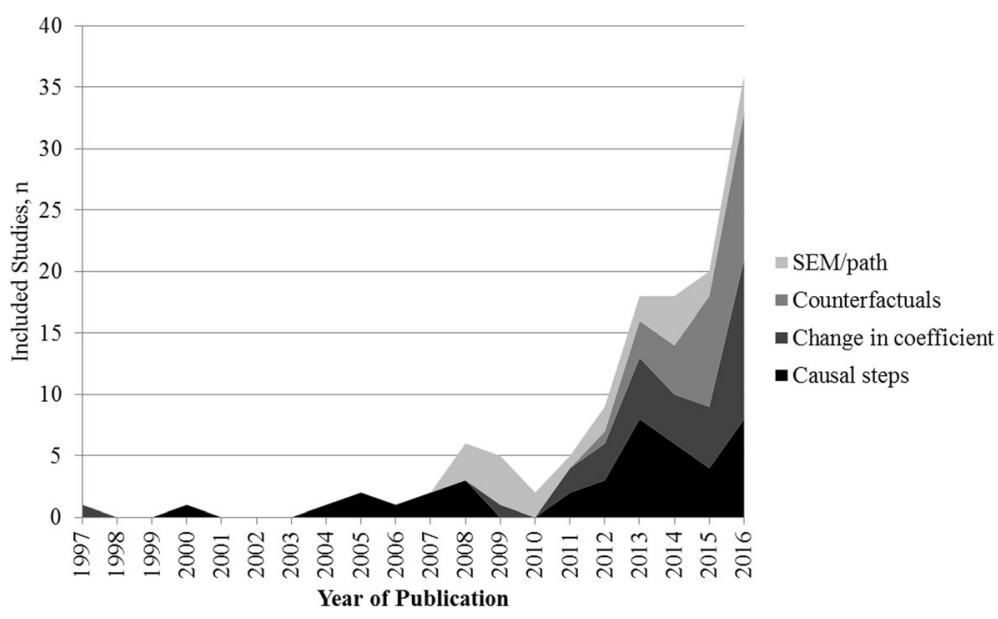

Fig. 5 Included studies by year, according to their approach to mediation analysis

(26\%) did not report either of these. Most studies reporting an indirect effect size included a measure of uncertainty ( $n=53$ of $55,96 \%)$ around the estimate; however, only 36 studies (of 83,43\%) included a measure of uncertainty around the proportion mediated. A total of 16 studies (11\%) included a test $p$-value, for the null hypothesis of no mediation.

The time-to-event outcome was most commonly modelled using a Cox PH model $(n=114,77 \%)$. Only $7 \%(n=8)$ of these included any mention of the rare outcomes assumption underlying use of this model. Of the 55 studies with a Cox PH model and an outcome frequency greater than $10 \%, 33(60 \%)$ reported an estimate for either the indirect effect or the proportion mediated.

There were 49 studies published from 1997 to 2012, and 100 studies published from 2013 to 2016. More recently published studies were more likely to include measures of the indirect effect or proportion mediated $(80 \%$ vs $63 \%, p=0.03)$, a measure of precision such as a $p$-value or $95 \%$ CI (69\% vs $45 \%, p=0.005)$, and a sensitivity analysis relating to mediation $(21 \%$ vs $8 \%, p=0.049$ ). In contrast, more recently published studies were not significantly more likely to contain mention of any ( $66 \%$ vs $63 \%, p=0.7)$ or all assumptions ( $8 \%$ vs $0 \%, p=0.053)$ underlying causal mediation analysis.

\section{Discussion}

We studied the use and reporting of mediation analysis with a time-to-event outcome in healthcare research. We found that the use of mediation analysis with time-to-event outcomes increased over time and crossed multiple clinical fields. The most common time-to-event outcomes were the onset or exacerbation of a medical condition, and the most common mediators were physiologic, psychological or lifestyle factors. This suggests that researchers are most interested in understanding whether specific patient-related factors explain disease onset. Although included studies were a mix of exploratory and confirmatory/hypothesis-based, over half of included studies did not have mediation analysis as the primary aim. This indicates that mediation analysis is often used as an adjunct to help understand the findings of a primary research question. There were several instances of repeated authorship. This suggests further mechanistic exploration following an early discovery (for example, the research into premature death in the visually impaired, by Christ, Zheng, Lee and Lam [40-43]) as well as spread of the tools of mediation by a few highly collaborative methodological experts.

Included healthcare studies covered a broad range of mediation analysis practices. The majority of mediation analyses were undertaken using traditional methods (change in coefficient or causal steps). While the publication of seminal methodological articles in 2010-2012 can explain the growth in the number of studies using a counterfactual approach, the concurrent rise in use of traditional approaches suggests heightened awareness of broad mediation concepts among clinical researchers. Many researchers may prefer traditional approaches due to their intuitive appeal and easy implementation.

A minority of studies reported or discussed the assumptions underlying causal interpretations of mediation analysis, as described by others [7]. Many studies measured exposures and mediators simultaneously at baseline. When the mediator does not occur after the exposure, this weakens the argument for causation. Few studies mentioned assumptions relating to confounding, or accurate measurement of the mediator. When underlying assumptions go unmentioned, readers may mistakenly believe causal conclusions to be more robust than they actually are. 
Most studies in our review used $\mathrm{Cox} \mathrm{PH}$ regression to model a time-to-event outcome. In such cases, obtaining an estimate of the indirect effect depends on the outcome being rare. Where the outcome is common, measures of the indirect effect or proportion mediated will be incorrect [20]. Yet, Cox Proportional Hazards were often used to model a common outcome, and nearly two thirds of such studies reported one or both of these measures. Further, the rare outcome assumption was infrequently mentioned.

Our study identifies further details on current research practices. While regression methods were frequently used to adjust for baseline characteristics (potential confounders), few studies included any form of sensitivity analysis relating to mediation. Interaction of the exposure and mediator was most often not considered. A minority of studies addressed competing risks, which alter the interpretation of mediated effects where the outcome is other than all-cause mortality. Specifically, reported effects are only valid for the population that remains alive. Although sample sizes were generally large, only one study attempted to justify sample size, despite the existence of programs designed for this purpose [44, 45].

Although recent studies were more likely to include effect sizes, measures of precision ( $p$-values or confidence intervals) and sensitivity analyses, reporting of characteristics and results of mediation analyses was overall suboptimal. The deficiencies identified in our study underscore the importance of developing standard reporting criteria for mediation analysis. Although others have made recommendations, no formal criteria have been published [7]. In addition to meeting established criteria for observational studies [35], we recommend that studies of mediation report the following items (see Table 3): whether mediation analysis is exploratory or confirmatory/hypothesis-based; the criteria used to assess mediation; the timing, measurement, and specification of exposure, mediator(s) and outcome variables; the type of model(s) and statistical software used; and methods used to account for any clustering or interactions between exposure and mediator. In addition, results reported should be accompanied by measures of precision ( $95 \%$ confidence intervals). Interpretation of the mediated effect should be made in the context of any competing risks (e.g. cause-specific indirect effect, among those who have not yet been censored). Assumptions underlying mediation analysis, and strategies used (regression, propensity scores, sensitivity analysis) to meet or test those assumptions should be detailed [9, 15]. Finally, the extent to which such assumptions limit causal inferences should be discussed in the limitations section.

We further recommend that researchers seeking to measure the degree of mediation or indirect effects avoid using a Cox PH model when the outcome is common (occurs in more than $10 \%$ of subjects). We suggest employing a counterfactual-based approach, which allows for mediators and outcomes of varied data distribution. Within this framework, the scale on which mediation is measured (hazard ratios, hazard differences) should be dictated by

Table 3 Reporting recommendations for mediation analysis with a time-to-event outcome

\begin{tabular}{|c|c|}
\hline Section & Recommendation \\
\hline Objectives & State whether mediation analysis(es) is/are exploratory or hypothesis-based \\
\hline \multirow[t]{8}{*}{ Methods } & $\begin{array}{l}\text { Specify criteria or statistical tests used to assess mediation, with references } \\
\text { Was the goal to categorize mediation as absent, partial or complete, or to estimate exact values for direct and } \\
\text { indirect effects? }\end{array}$ \\
\hline & Detail how exposure, mediator and outcome variables were defined and measured \\
\hline & Detail when exposure, mediator and outcome variables were measured \\
\hline & $\begin{array}{l}\text { Describe statistical models used for the mediator(s) and outcome(s), and any assumptions underlying use of } \\
\text { such models (e.g. proportionality, rare outcome assumption for Cox Proportional Hazards models) }\end{array}$ \\
\hline & State whether interaction between exposure and mediator was considered, and how \\
\hline & Reference any software programs used for mediation analysis \\
\hline & $\begin{array}{l}\text { If relevant for exposure, mediator, and outcome being considered, state how the following were addressed: } \\
\text { - clustering or repeated events } \\
\text { - competing risks }\end{array}$ \\
\hline & $\begin{array}{l}\text { Describe assumptions underlying mediation analysis, and methods used to address these (e.g.: randomisation, } \\
\text { regression, weighting, stratification, sensitivity analysis) }\end{array}$ \\
\hline \multirow[t]{2}{*}{ Results } & $\begin{array}{l}\text { Report measures of mediation effect (indirect effect or proportion mediated) accompanied by } 95 \% \text { confidence } \\
\text { intervals }\end{array}$ \\
\hline & Report p-values for mediation hypothesis testing \\
\hline Discussion & $\begin{array}{l}\text { Discuss limitations of causal inference based on mediation analysis results, including whether underlying } \\
\text { assumptions were met } \\
\text { Discuss magnitude and direction of any potential bias }\end{array}$ \\
\hline
\end{tabular}

In addition to these, mediation analyses should meet the STROBE criteria for observational studies [35] 
the medical problem at hand. Marginal structural, additive hazards and parametric survival models can be used when the outcome is common ( $>10 \%)$. If familiarity and ease of implementation strongly favours a Cox-based approach, then authors must confirm that the outcome is rare.

Strengths of our study include its systematic search of multiple databases, and pre-defined extraction criteria. Further, previous systematic reviews of mediation analysis have been limited to specific journals or studies published in 2015 [6, 7]. While we were focused on mediation analysis with a time-to-event outcome, our inclusion of all methodological approaches over a long time frame has better illustrated the evolution of real-world research practices with this emerging methodology.

This study has several limitations. First, mediation analysis and time-to-event did not have specific index terms available in the databases searched, and thus we relied on keyword searching to identify eligible studies. We mitigated this by using a broad range of terms to maximize sensitivity. Second, our findings are limited to published studies. However, this was intentional as we were interested in understanding which practices would be accepted in the peer-reviewed literature. Third, it is possible that authors are not reporting their full approach to mediation analysis due to space limitations. This underscores the need for standard reporting criteria, in order to help authors, reviewers, and editors prioritize content.

\section{Conclusions}

Mediation analysis for time-to-event outcomes is being used with increasing frequency by researchers around the world. There is ongoing reliance on traditional methods such as causal steps and change in coefficient. When combined with Cox PH modelling, these methods are limited to use with rare outcomes. As a result, methods using counterfactuals and/or alternative survival models are preferred. We provide preliminary criteria that may be used by researchers reporting or reviewing similar studies. However, as mediation analysis is increasingly used in clinical research, a comprehensive set of reporting criteria must be more formally developed, with input from clinicians, healthcare researchers, journal editors and methodological experts. Such criteria will greatly benefit researchers seeking to report not only the "why" but also the "how" of their findings.

\section{Additional files}

Additional file 1: Comprehensive search strategy. Description: this file contains the search terms and strategy using in our comprehensive search. (DOCX $21 \mathrm{~kb}$ )

Additional file 2: Included Studies. Description: this table contains the details of all published papers selected for study inclusion. (DOCX 42 kb)

\section{Abbreviations}

Cox PH: Cox Proportional Hazards; SEM: Structural Equation Modelling

\section{Acknowledgements}

Not applicable.

\section{Funding}

This project did not receive any specific funding. LLS is supported by a CIHR Fellowship Award (FRN 146714) and the Philipson Scholar program at the University of Toronto.

\section{Availability of data and materials}

The datasets used and/or analysed during the current study are available from the corresponding author on reasonable request.

\section{Authors' contributions}

LLS TL AOC PCA NMI DAR CMB designed the study, LLS ZB AOC performed data collection, LLS NH performed data analysis. LLS drafted the manuscript, and all authors revised it critically for important intellectual content. All authors approve of the final submitted manuscript.

Ethics approval and consent to participate Not applicable.

\section{Consent for publication}

Not applicable.

\section{Competing interests}

$\mathrm{TL}$ reports personal fees from Novo Nordisk and BeiGene, outside the submitted work. The remaining authors declare that they have no competing interests.

\section{Publisher's Note}

Springer Nature remains neutral with regard to jurisdictional claims in published maps and institutional affiliations.

\section{Author details}

${ }^{1}$ Department of Medicine, University of Toronto, Toronto, Canada. ${ }^{2}$ Institute for Health Policy, Management and Evaluation, University of Toronto, Toronto, Canada. ${ }^{3}$ Institute for Clinical Evaluative Sciences, Toronto, Canada. ${ }^{4}$ Institute for Health Systems Solutions and Virtual Care, Women's College Hospital, Toronto, Canada. ${ }^{5}$ Centre for Urban Health Solutions, St. Michael's Hospital, Toronto, Canada. ${ }^{6}$ Section of Biostatistics, University of Copenhagen, Copenhagen, Denmark. ${ }^{7}$ Center for Statistical Science, Peking University, Beijing, China. ${ }^{8}$ Library and Information Services, University Health Network, Toronto, Canada. ${ }^{9}$ Department of Family and Community Medicine, University of Toronto, Toronto, Canada. ${ }^{10}$ Department of Medicine, Mount Sinai Health System, Toronto, Canada.

Received: 16 February 2018 Accepted: 16 October 2018

Published online: 29 October 2018

\section{References}

1. MacKinnon DP, Fairchild AJ, Fritz MS. Mediation analysis. Annu Rev Psychol. 2007:58:593-614.

2. Valeri L, Vanderweele TJ. Mediation analysis allowing for exposure-mediator interactions and causal interpretation: theoretical assumptions and implementation with SAS and SPSS macros.[Erratum appears in Psychol Methods. 2013 Dec;18(4):474]. Psychol Methods. 2013;18(2):137-50.

3. Lange T, Rasmussen M, Thygesen LC. Assessing natural direct and indirect effects through multiple pathways. Am J Epidemiol. 2014;179(4):513-8.

4. Fairchild AJ, Mackinnon DP. A general model for testing mediation and moderation effects. Prev Sci. 2009;10(2):87-99.

5. Preacher KJ, Rucker DD, Hayes AF. Addressing moderated mediation hypotheses: theory, methods, and prescriptions. Multivar Behav Res. 2007;42(1):185-227

6. Lange T, Starkopf L. Commentary: mediation analyses in the real world. Epidemiology. 2016;27(5):677-81.

7. Liu S-H, Ulbricht CM, Chrysanthopoulou SA, Lapane KL. Implementation and reporting of causal mediation analysis in 2015: a systematic review in epidemiological studies. BMC Res Notes. 2016;9:354. 
8. Imai K, Keele L, Tingley D. A general approach to causal mediation analysis. Psychol Methods. 2010;15(4):309-34.

9. VanderWeele TJ. Bias formulas for sensitivity analysis for direct and indirect effects. Epidemiology. 2010;21(4):540-51.

10. VanderWeele T, Vansteelandt $\mathrm{S}$. Conceptual issues concerning mediation, interventions and composition. Statistics Interface. 2009;2:457-68.

11. Richiardi L, Bellocco R, Zugna D. Mediation analysis in epidemiology: methods, interpretation and bias. Int J Epidemiol. 2013;42(5):1511-9.

12. Small DS. Mediation analysis without sequential Ignorability: using baseline covariates interacted with random assignment as instrumental variables. Philadelphia: University of Pennsylvania; 2016.

13. VanderWeele TJ, Valeri L, Ogburn EL. The role of measurement error and misclassification in mediation analysis: mediation and measurement error. Epidemiology. 2012;23(4):561-4.

14. Gelfand LA, MacKinnon DP, DeRubeis RJ, Baraldi AN. Mediation analysis with survival outcomes: accelerated failure time vs. proportional hazards models. Front Psychol. 2016;7:423.

15. Preacher KJ. Advances in mediation analysis: a survey and synthesis of new developments. In: Fiske ST, editor. Annual Review of Psychology, Vol 66. Volume 66; 2015. p. 825-52.

16. Baron RM, Kenny DA. The moderator-mediator variable distinction in social psychological research: conceptual, strategic, and statistical considerations. J Pers Soc Psychol. 1986;51(6):1173-82.

17. Fritz MS, Mackinnon DP. Required sample size to detect the mediated effect. Psychol Sci. 2007;18(3):233-9.

18. MacKinnon DP, Lockwood CM, Hoffman JM, West SG, Sheets V. A comparison of methods to test mediation and other intervening variable effects. Psychol Methods. 2002;7(1):83-104.

19. Di Serio C, Rinott Y, Scarsini M. Simpson's paradox in survival models. Scand J Stat. 2009;36(3):463-80.

20. Sjolander A, Dahlqwist E, Zetterqvist J. A note on the noncollapsibility of rate differences and rate ratios. Epidemiology. 2016;27(3):356-9.

21. VanderWeele TJ. Causal mediation analysis with survival data. Epidemiology. 2011;22(4):582-5.

22. Martinussen T, Vansteelandt S. On collapsibility and confounding bias in cox and Aalen regression models. Lifetime Data Anal. 2013;19(3):279-96.

23. Muthen B, Asparouhov T. Causal effects in mediation modeling: an introduction with applications to latent variables. Struct Equ Model Multidiscip J. 2015;22(1):12-23.

24. Pearl J. The causal mediation formula - a guide to the assessment of pathways and mechanisms. Prev Sci. 2012;13(4):426-36.

25. Pratschke J, Haase T, Comber H, Sharp L, Cancela MD, Johnson H. Mechanisms and mediation in survival analysis: towards an integrated analytical framework. BMC Med Res Methodol. 2016;16.

26. Muthén B, Masyn K. Discrete-time survival mixture analysis. J Educ Behav Stat. 2005;30(1):27-58.

27. Singer JD, Willett JB. It's about time: using discrete-time survival analysis to study duration and the timing of events. J Educ Stat. 1993;18(2):155-95.

28. Fosen J, Ferkingstad E, Borgan O, Aalen OO. Dynamic path analysis-a new approach to analyzing time-dependent covariates. Lifetime Data Anal. 2006;12(2):143-67.

29. VanderWeele TJ. Mediation analysis: a Practitioner's guide. In: Fielding JE, editor. Ann Rev Public Health. 2016;37:17-32.

30. Rubin D. Estimating causal effects of treatments in experimental and observational studies. ETS Research Bulletin Series. 1972:1972(2):i-31.

31. Tchetgen EJT. On causal mediation analysis with a survival outcome. Int J Biostat. 2011;7(1).

32. Lange T, Hansen JV. Direct and indirect effects in a survival context. Epidemiology. 2011;22(4):575-81.

33. Lange T, Vansteelandt S, Bekaert M. A simple unified approach for estimating natural direct and indirect effects. Am J Epidemiol. 2012;176(3):190-5.

34. Valeri L, VanderWeele TJ. SAS macro for causal mediation analysis with survival data. Epidemiology. 2015;26(2):e23-4.

35. Elm Ev ADG, Egger M, Pocock SJ, Gøtzsche PC, Vandenbroucke JP. Strengthening the reporting of observational studies in epidemiology (STROBE) statement: guidelines for reporting observational studies. BMJ. 2007;335(7624):806-8.

36. Lee H, Hubscher M, Moseley GL, Kamper SJ, Traeger AC, Mansell G, McAuley $J$ H. How does pain lead to disability? A systematic review and meta-analysis of mediation studies in people with back and neck pain. Pain. 2015;156(6):988-97.
37. Gamer ML J, Fellows I, Singh P.: irr: Various Coefficients of Interrater Reliability and Agreement. In.: R package version 0.84.; 2012.

38. Clarivate Analytics: 2016 Journal Citation Reports; 2017.

39. Hassan M, Qureshi W, Sroujieh LS, Albashaireh D, BouMalham S, Liroff M, Amjad W, Khalid F, Hadid H, Alirhayim Z. Interplay of parathyroid hormone and aldosterone antagonist in prevention of heart failure hospitalizations in chronic kidney disease. J Renin-Angiotensin-Aldosterone Syst. 2014;15(3): 278-85.

40. Christ SL, Zheng D, Swenor BK, Lam BL, West SK, Tannenbaum SL, Munoz $\mathrm{BE}$, Lee DJ. Longitudinal relationships among visual acuity, daily functional status, and mortality the salisbury eye evaluation study. JAMA Ophthalmol. 2014;132(12):1400-6.

41. Lam BL, Christ SL, Lee DJ, Zheng DD, Arheart KL. Reported visual impairment and risk of suicide: the 1986-1996 national health interview surveys. Arch Ophthalmol. 2008;126(7):975-80.

42. Zheng DD, Christ SL, Lam BL, Arheart KL, Galor A, Lee DJ. Increased mortality risk among the visually impaired: the roles of mental well-being and preventive care practices. Invest Ophthalmol Vis Sci. 2012;53(6):2685-92.

43. Zheng DD, Christ SL, Lam BL, Tannenbaum SL, Bokman CL, Arheart KL, McClure LA, Fernandez CA, Lee DJ. Visual acuity and increased mortality: the role of allostatic load and functional status. Invest Ophthalmol Vis Sci. 2014:55(8):5144-50

44. Vittinghoff $E$, Neilands TB. Sample size for joint testing of indirect effects. Prev Sci. 2015;16(8):1128-35.

45. Vittinghoff E, Sen S, McCulloch CE. Sample size calculations for evaluating mediation. Stat Med. 2009;28(4):541-57.

\section{Ready to submit your research? Choose BMC and benefit from:}

- fast, convenient online submission

- thorough peer review by experienced researchers in your field

- rapid publication on acceptance

- support for research data, including large and complex data types

- gold Open Access which fosters wider collaboration and increased citations

- maximum visibility for your research: over $100 \mathrm{M}$ website views per year

At BMC, research is always in progress.

Learn more biomedcentral.com/submissions 